\title{
Porous Poly(3-hydroxybutyrate) Scaffolds Prepared by Non-Solvent- Induced Phase Separation for Tissue Engineering
}

\author{
Jiseon Kang ${ }^{1}$ \\ Ji-Young Hwang ${ }^{2}$ \\ Mongyoung $\mathrm{Huh}^{2}$ \\ Seok Il Yun ${ }^{* 1}$
}

\author{
${ }^{1}$ Department of Chemical Engineering and Materials Science, Sangmyung University, Seoul 03016, Korea \\ ${ }^{2}$ Korea Institute of Carbon Convergence Technology, Jeonju-si, Jeonbuk 54853, Korea
}

Received December 7, 2019 / Revised April 7, 2020 / Accepted April 8, 2020

\begin{abstract}
Highly porous poly(3-hydroxybutyrate) (PHB) scaffolds were fabricated using non-solvent-induced phase separation with chloroform as the solvent and tetrahydrofuran as the non-solvent. The microporosity, nanofiber morphology, and mechanical strength of the scaffolds were adjusted by varying the fabrication parameters, such as the polymer concentration and solvent composition. The influence of these parameters on the structure and morphology of PHB organogels and scaffolds was elucidated using small-angle neutron scattering and scanning electron microscopy. The organogels and scaffolds in this study have a complex hierarchical structure, extending over a wide range of length scales. In vitro viability assays were performed using the human keratinocyte cell line (HaCaT), and all PHB scaffolds demonstrated the excellent cell viability. Microporosity had the greatest impact on HaCaT cell proliferation on PHB scaffolds, which was determined after a 3-day incubation period with scaffolds of different morphologies and mechanical properties. The superior cell viability and the controlled scaffold properties and morphologies suggested PHB scaffolds fabricated by non-solvent-induced phase separation using chloroform and tetrahydrofuran as promising biomaterials for the applications of tissue engineering, particularly of epidermal engineering.
\end{abstract}

Keywords: PHB, non-solvent-induced phase separation, scaffold, cell viability, micropores, nanofibers.

\section{Introduction}

The phase-separation method is one of the most powerful methods used to fabricate porous scaffolds. By mixing an appropriate non-solvent into a homogeneous polymer solution, the polymer-solvent affinity decreases to induce a liquid-liquid phase separation. ${ }^{1-10}$ This forms an interpenetrating network of a polymer-rich phase and a polymer-poor phase referred to as nonsolvent-induced phase separation (NIPS). Liquid-liquid phase separation of a polymer solution can be induced, by non-solvent addition and by changing temperature. This is referred as temperature-induced phase separation (TIPS). ${ }^{11-26}$ Theoretically, a polymer-rich phase consists of the polymer and a fraction of the solvent. A polymer-poor phase contains a non-solvent and the remaining solvent. For semi-crystalline polymers, crystallization occurs in the polymer-rich phase. As a result, the solution forms a gel as microcrystallites serve as junctions for a three dimensional (3-D) network ${ }^{1-4,8,24-26}$ The polymer-rich phase develops the skeleton of the gel monolith, whereas the polymer-poor phase flows through the matrix, resulting in porous channels within the monolith. These organogels can become high-quality scaffolds with high porosity after careful removal of the solvents. Furthermore, the unique nanofibrous structure can be produced by suitably manipulating processing conditions of phase separation and drying procedures. ${ }^{10,12,27}$ Poly(3-hydroxybutyrate) (PHB)

*Corresponding Author: Seok Il Yun (yunsans@smu.ac.kr) is a semi-crystalline, linear polyester synthesized by microorganisms. It receives considerable attention in the literature, owing to its excellent biodegradability, biocompatibility, and mechnaical strength, suitable for scaffold materials within tissue engineering. ${ }^{28-30} \mathrm{PHB}$ has shown promise as scaffolding material for the regeneration of various tissues including cardiovascular and heart valve, nerve conduit, cartilage, liver, skin and bone tissues. ${ }^{29,30}$ However, for tissue engineering applications, the physical and mechanical properties of PHB need to be improved. The main limitation of PHB is the lack of hydrophilicity and bioactivity. PHB composites with hydrophilic and bioactive modifiers have been intensively investigated to overcome these problems. In particular, the addition of bioceramics such as hydroxyapatite and bioglass to the PHB matrix has been shown to improve biological activity and the mechanical properties of PHB scaffolds. This has resulted in enhanced cell attachment and proliferation for bone tissue engineering. ${ }^{31-33}$ In designing a tissue engineering scaffold, connected micropores play a major role in triggering the regeneration of new tissues. The simplest method to develop macroporous matrices is the particulate-leaching method involving the leaching of soluble particulates or porogens. In comparison with scaffolds produced by the particulate-leaching method, scaffolds produced by TIPS show higher mechanical strength, while maintaining the same porosity degree. Tissue engineering scaffolds made of PHB and its copolymers with well-connected micropores have previously been prepared by TIPS. ${ }^{10-16}$ NIPS have also been applied to produce highly porous 
and nanofibrous PHB scaffolds using chloroform (CF) as the solvent and tetrahydrofuran (THF) as the non-solvent. ${ }^{10,12}$ It has been shown that the morphology and properties of PHB scaffolds can be modulated by varying the solvent composition (i.e., the THF/CF volume ratio). ${ }^{10}$ However, the relationship between cell viability, scaffold morphology and properties has not previously been tested. In addition, one of the most important parameters, the polymer concentration effect on the scaffold structures, has been neglected in previous studies. ${ }^{10}$ To date, quantitative structural characterization of PHB gels prepared by NIPS or TIPS has only been performed for crystallization behavior. ${ }^{10,11}$ The current study deals with the quantative analysis of the multiscale structure of PHB organogels and scaffolds, from the nanoto micro-range, by small-angle neutron scattering (SANS). The effects of polymer concentration on the morphology and properties of the PHB scaffolds manufactured by NIPS was examined, providing a more complete understanding of the relationship between fabrication parameters and scaffold properties. Finally, the in vitro biocompatibility was characterized using the human keratinocytes cell line (HaCaT) to assess the potential for PHB scaffolds to be used in tissue engineering applications. Compared to numerous studies on various cells for bone and cardiovascular tissues, relatively little research has been undertaked using PHB and its copolymers to support HaCaT cell growth for skin tissue engineering. ${ }^{12,29,34-36}$ Excellent control of the scaffold structure by NIPS makes it possible to compare cell proliferation on PHB scaffolds with various morphology and mechanical properties. Microporosity has been found to have the largest impact on HaCaT cell proliferation on PHB scaffolds.

\section{Experimental}

\subsection{Materials and fabrication of PHB scaffolds}

Materials and preparation methods for PHB organogels and scaffolds are discussed in detail elsewhere. ${ }^{10}$ Briefly, the PHB solutions were prepared by dissolving PHB polymer $\left(M_{\mathrm{w}}=4.37 \times 10^{5}\right.$, Sigma-Aldrich) in $\mathrm{CF}$ under stirring at $95^{\circ} \mathrm{C}$ then cooling the solutions down to $25^{\circ} \mathrm{C}$. The THF, was then added to the PHB solutions at room temperature. The mixed solutions were kept at $25^{\circ} \mathrm{C}$ using heating blocks during the phase separation followed by gelation. The PHB concentration was defined on the basis of the total volume of chloroform and THF. The ratio of nonsolvent/solvent (THF/CF v/v) was varied from 30/70 to 60/40. The PHB concentration ( $\phi$ ) were adjusted from 0.005 to $0.03 \mathrm{~g} / \mathrm{mL}$. Gelation was confirmed by the formation of self-supporting samples that did not flow when inverted $180^{\circ}$. The gel transition time determined by the inversion tests depended on the solvent composition and PHB concentration. The wet, aged gels at $25^{\circ} \mathrm{C}$ for two weeks were removed by breaking their glass vessels and were immersed in methanol. The solvent (CF) and non-solvent (THF) of the monolithic organogels were sequentially extracted by soaking the gel first in methanol for 2 days and then in water at room temperature for 2 days. Finally, the as-produced organogels were freeze-dried under 30 mTorr at $130{ }^{\circ} \mathrm{C}$ for 2 days using an FDCF- 12003 freeze dryer (Operon, Korea) to produce porous PHB scaffolds.

\subsection{Characterization}

The structure of the PHB organogels and scaffolds was characterized by SANS. SANS measurements were performed on the QUOKKA beamline at ANSTO (Sydney, Australia), using three sample-to-detector distances $(2,14$, and $20 \mathrm{~m})$ and wavelengths of 5 and $8 \AA^{37}$ The three configurations covered a $Q$ range from $8.8 \times 10^{-4}$ to $0.33 \AA^{-1}$. The raw data was corrected for scattering from the empty cell, detector dark current, detector sensitivity, sample transmission, and thickness. Following these corrections, the data was placed on an absolute scale using a calibrated secondary standard and circularly averaged to produce $I(Q)$ versus $Q$ plots, where $I(Q)$ is the scattered intensity and $Q=$ $\sin (\theta) 4 \pi / \lambda$ is the scattering vector for a scattering angle, $\theta$. Data analysis was based on fitting the scattering curve to an appropriate model using software provided by the National Institute of Standards and Technology (NIST, SANS analysis version 7.0 on IGOR) ${ }^{38}$ To prepare PHB organogels for SANS measurements, deuterated CF was used as the solvent. THF and deuterated THF were mixed to match the scattering length density of deuterated CF to eliminate the heterogeneity originated from non-uniform solvent distribution.

Scaffold morphology was obtained by scanning electron microscopy (SEM) (JSM-6701F, JEOL Ltd, Japan) at an accelerated voltage of 5-15 kV. A thin layer of platinum (approximately several nanometers) was sputter-coated before scanning. The surface area of the scaffolds was calculated by low temperature $\mathrm{N}_{2}$ adsorption-desorption (Micromeritics ASAP 2000MP) using the BrunauerEmmett-Teller (BET) equation. The compressive stress-strain curves of the cylindrical scaffolds $(12 \mathrm{~mm}$ in diameter and 10 $\mathrm{mm}$ in height) were obtained by a Universal Testing Machine (Instron 5543) using a crosshead speed of $1 \mathrm{~mm} / \mathrm{min}$ and a static load of $1 \mathrm{kN}$. The compressive moduli were calculated from the slope of the initial linear region of the stress-strain curves. Three replicate specimens were run for each sample, and the results are provided as the average value \pm standard deviation. The melting behavior of the PHB crystalline phase was analyzed for the as-prepared PHB scaffolds by differential scanning calorimetry (DSC, Discovery DSC: TA instrument, USA) in a temperature range from 20 to $200^{\circ} \mathrm{C}$. The apparent density, $\rho_{\text {aeroge, }}$ of the scaffolds was calculated from the mass and volume of the dried PHB scaffolds. The overall porosity was determined from the dry mass and volume of the scaffolds using

Porosity $(\%)=1-\frac{\rho_{\text {aerogel }}}{\rho_{\mathrm{PHB}}} \times 100$

where $\rho_{\mathrm{PHB}}$ is the density of non-porous raw PHB, $\rho_{\mathrm{PHB}}=1.260 \mathrm{~g} /$ $\mathrm{cm}^{3}{ }^{12}$

The human keratinocytes cell line (HaCaT) was cultured in tissue culture flasks using Dulbecco's modified Eagle's medium (DMEM) containing 10\% (v/v) fetal bovine serum, $1 \%$ penicillin, and $100 \mathrm{~g} / \mathrm{mL}$ streptomycin (Gibco, USA) at $37^{\circ} \mathrm{C}$ in a $5 \% \mathrm{CO}_{2}$ incubator (Heracell VIOS 160i, Thermo Fisher Scientific, USA). Each scaffold was first cut into $2 \mathrm{~mm}$ thick slices, placed in 24well plates on double-sided adhesive tape, washed with $70 \%$ ethanol, and then followed by sterilization by ultraviolet (UV) irradiation for $30 \mathrm{~min}$. The sterilized PHB was immersed in cul- 
ture medium for several hours until the scaffolds were wellhydrated, and washed with culture medium three times before the cells were seeded. HaCat cells were seeded onto each PHB scaffold and placed in a 96-well plate at a density of $1 \times 10^{4}$ cells per well and incubated for 3 days. The cell viability of HaCaT cells on PHB was determined using a Cell Counting Kit-8 assay (CCK-8; Dojindo Laboratories, Japan), according to the manufacturer's instructions.

To investigate cell morphology, HaCaT cells seeded on the scaffolds were fixed with $2.5 \%$ glutaraldehyde, dehydrated through a series of ethanol concentrations (50\%, 75\%, 95\%, and 100\%), treated with hexamethyldisilazane, and then dried in the hood overnight. The surface morphology of the HaCaT cells on the scaffolds was observed using a GeminiSEM 500 field emission SEM (Zeiss, Germany) operated at an accelerating voltage of $3.0 \mathrm{kV}$ by standard procedures. Actin filaments in the cytoskeleton and nucleus of HaCaT cells on scaffolds were stained with Alexa Fluor 488 phalloidin and 4',6-diamidino-2-phenylindole (DAPI), respectively, according to the manufacturer's instructions (Thermo Fisher Scientific, USA), followed by imaging the cells under a fluorescence microscope (Zeiss, Germany). All experiments were repeated three to six times, and data are presented as the means \pm standard deviations. Statistical analysis was performed using GraphPad Prism (GraphPad software, USA). Statistical significance of between-group differences was evaluated with one-way ANOVA. Differences were considered statistically significant at $p<0.05$.

\section{Results and discussion}

\subsection{SANS analysis}

PHB organogels with varying polymer concentrations and solvent compositions were prepared. Gelation of PHB solutions in $\mathrm{CF}$ was achieved at room temperature by the addition of THF. Gelation was confirmed by the formation of self-supporting samples that did not flow when inverted at $180^{\circ}$ (Figure S1). The PHB organogels were characterized using SANS. Small-angle $\mathrm{x}$-ray scattering (SAXS) and SANS have been successfully used to study the hierarchical structure of gels induced by a liquidliquid phase separation. ${ }^{39-41}$ Figure 1 shows log-log plots of SANS intensity versus momentum transfer $(Q)$ as a function of THF/ $\mathrm{CF}$ ratios and $\phi$. All SANS profiles clearly show an upturn at low angle, suggesting the presence of large inhomogeneities in the gel structure caused by phase separation. The upturn scattering at low $Q$ follows a power law,

$$
I(Q) \sim Q^{-n}
$$

The exponent $(n)$ characterizes the fractal structure of the longrange frozen inhomogeneities at low $Q^{42}$ The $n$ value is related to either the mass $\left(D_{m}\right)$ or the surface $\left(D_{s}\right)$ fractal dimension. When $n<3$, the scattering is due to a mass fractal structure, and $n=D_{m}$. For a surface fractal structure, $3<n<4$ and $n=6-D_{s} . D_{m}$ indicates the "openness" of the fractal dimension. $D_{m}$ equals 1 for loosely connected aggregates possessing indistinct interfaces, and 3 for dense aggregates. $D_{s}$ is 3 for rough surfaces and 2 for smooth surfaces. The organogels have $n$ values between 2 and 3, regardless of solvent compositions and PHB concentrations, indicating that the boundary between polymer-rich and -poor phases is a mass fractal structure. For conventional polymer network structures at high $Q\left(>0.07 \mathrm{~A}^{-1}\right)$, the scattering intensity, $I(Q)$, typically decreases with increasing $Q$, which is well modelled by the Ornstein-Zernike (OZ) equation, ${ }^{43}$

$I(Q)=\frac{A}{\left(1+\xi^{2} Q^{2}\right)}+\mathrm{Bkg}$

where $Q$ is the scattering vector, $\xi$ is the correlation length, and Bkg is background scattering. However, for the PHB organogels described here, the OZ model failed to fit the data at high $Q$ $>0.07 \AA^{-1}$. Interestingly, the scattering intensity decreases with $Q^{-4}$ for all concentrations (Figure 1 ). This $Q$ dependence corresponds with Porod's law, indicating that PHB forms small crystallites during gelation, and the surfaces of the PHB crystallites have a clear boundary. Semi-crystalline polymers form nanocrystalline structures in polymer-rich phases during phase separation, which act as a physical cross-linker in forming a polymer 3-D network (Figure 2). The formation of a PHB crystalline phase during phase separation was previously measured in situ using FTIR. ${ }^{10}$ Display of 'Porod's law' behavior at higher $Q$ has been reported for PVA gels prepared by phase separation due to the formation of small crystalline phases. ${ }^{39-41}$ It has been suggested that the $I(Q)$ profiles in the transition regions, between the range of the two power laws at high and low $Q$, originates from the correlation between cross-linking points (crystallites), and that the scattering profile can be described by the $\mathrm{OZ}$ formula (Eq. (3)). ${ }^{39-41}$ The correlation length, $\xi$, represents the range of the spatial correlation of concentration fluctuations in the system. In this case, $\xi$ obtained from fits to Eq. (3) represented either the mesh size of the PHB network or the average distance between the nearest-neighbor cross-linking points (crystallites). The solid line in Figure 1(A) denotes the best fit to the observed $I(Q)$ in the range $0.01<Q<0.035 \mathrm{~A}^{-1}$ using the $\mathrm{OZ}$ formula. The correlation lengths evaluated from the fits are plotted in Figure $3(\mathrm{~A})$ for organogels with varying THF $/ \mathrm{CF}$ ratios and at fixed $\phi(0.03 \mathrm{~g} / \mathrm{mL})$. The correlation length of the gel was found to increase with decreasing THF/CF ratio. Decreasing the THF/CF ratio (increas-
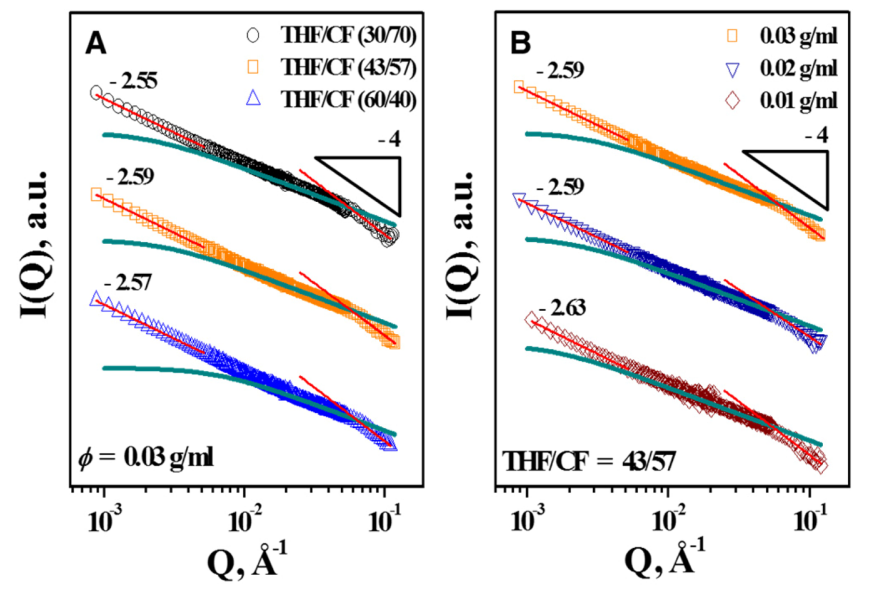

Figure 1. SANS data of PHB organogels with (A) varying THF/CF ratios and a fixed $\mathrm{PHB}$ concentration $(\phi, 0.03 \mathrm{~g} / \mathrm{mL})$, and (B) varying $\phi$ and a fixed THF/CF ratio (43/57). Curves are shifted vertically for clarity. 


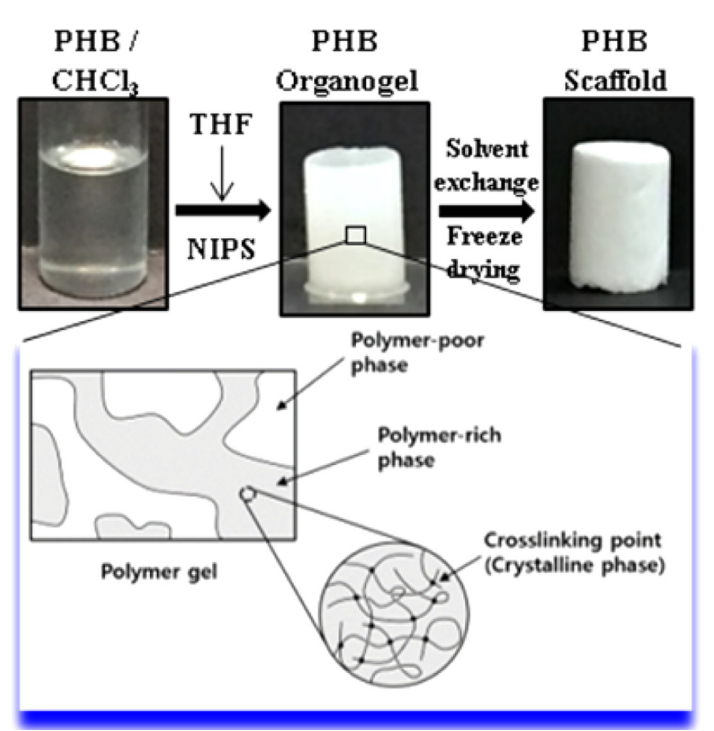

Figure 2. Schematic illustrations of the NIPS process and the organogel structure.

ing $(F)$ at fixed $\phi$ effectively decreased PHB concentration in the PHB-rich phase, resulting in an increase in $\xi$. SANS was performed on the PHB organogels as a function of $\phi$ at fixed THF/ CF ratio (43/57), as displayed in Figure 1(B). The $n$ values did not change significantly with $\phi$; however, $\xi$ was found to decrease with $\phi$, indicating that increasing $\phi$ results in more PHB in the PHB-rich phase (Figure 3(B)). The wet, aged organogels were transformed into aerogels (scaffolds) by soaking the gel first in methanol and then in water, followed by freeze drying. The structure of these scaffolds was characterized by SANS. Figure 4(A), (B) show the SANS curves of scaffolds with varying solvent compositions and at a constant $\phi$ of 0.02 and $0.03 \mathrm{~g} / \mathrm{mL}$, respectively. Although the organogels were transformed into aerogels by solvent exchange and freeze drying, $n$ values between 2 and 3 were still observed, which are characteristic of a mass fractal. This indicates that the interfacial structure between the two phases did not change during solvent exchange and freeze drying. Compared to the SANS curves of the organogels, the Porod's scattering at high $Q$ extended to lower $Q$, indicating that the crystalline phase became larger after the solvent exchange and freeze drying. The disappearance of the $\mathrm{OZ}$ region in the SANS

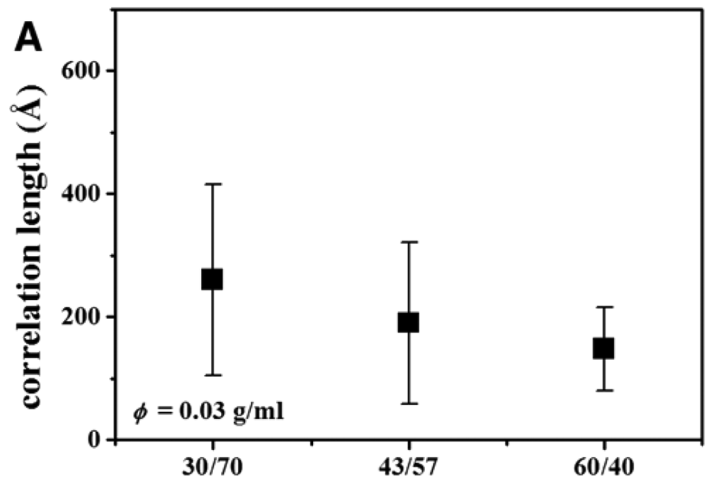

Solvent composition (THF/CF)
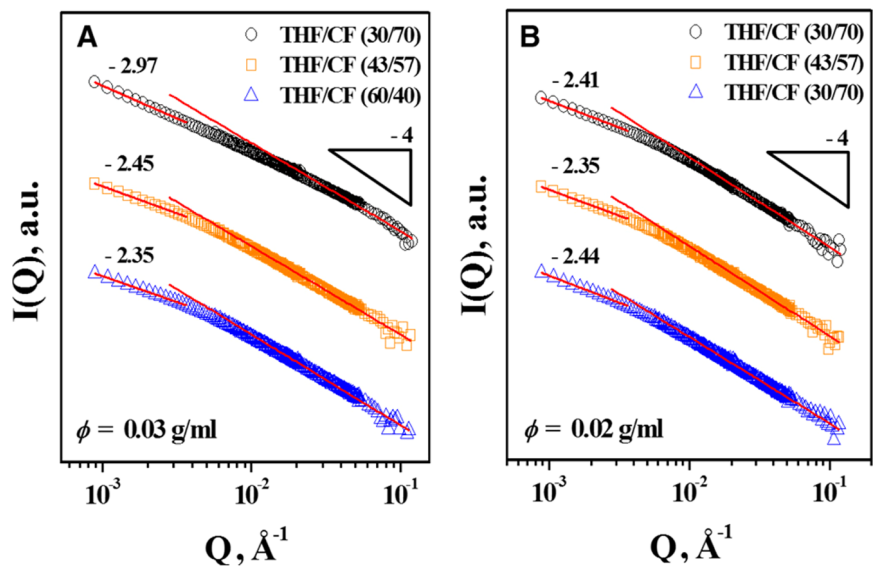

Figure 4. SANS curves of PHB scaffolds with varying solvent compositions and a fixed PHB concentration ( $\phi$ ) of (A) $0.02 \mathrm{~g} / \mathrm{mL}$ and (B) $0.03 \mathrm{~g} / \mathrm{mL}$. Curves are shifted vertically for clarity.

curves also implies that the polymer network structure embedded with small crystallites of the polymer-rich phase turned into a nanofibrous skeleton structure after the solvent exchange and freeze-drying process.

\subsection{SEM analysis}

Although the total porosity of the PHB scaffolds produced by NIPS did not change with solvent composition, the pore properties and morphology of PHB scaffolds varied greatly. The welldefined nanofibers changed into an almost non-fibrous skeleton surrounding well-connected micropores with increasing nonsolvent content (Figure 5(A), (I)). ${ }^{10}$ According to NIPS theory, the polymer-poor phase consists mostly of a non-solvent. A lower non-solvent fraction (THF/CF ratio of 30/70) can lead to a smaller or fewer polymer-poor regions, which uniformly interpenetrate the polymer-rich phases in the organogels, resulting in a welldefined nanofiber morphology of the resulting scaffold after solvent evaporation (Figure 5(A)). By increasing the THF/CF ratio to $60 / 40$ at fixed $\phi(0.03 \mathrm{~g} / \mathrm{mL})$, the nanofibrous morphology was suppressed substantially and the microporous morphology became dominant (Figure 5(I) $){ }^{10}$ SANS result shows that increasing the THF/CF ratio not only increases the size of the polymer-poor

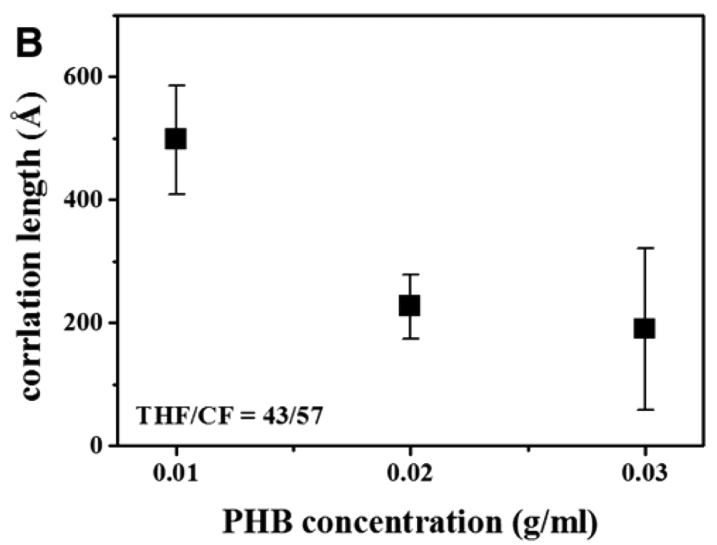

Figure 3. Correlation length as a function of (A) THF/CF ratio with a fixed PHB concentration $(\phi, 0.03 \mathrm{~g} / \mathrm{mL})$ or (B) $\phi$ with a fixed THF/CF ratio (43/57), obtained by fitting the mid- $Q$ range to the $\mathrm{OZ}$ equation. The relative uncertainties (error bars) shown here are the standard deviations, based on the goodness of the fit. 


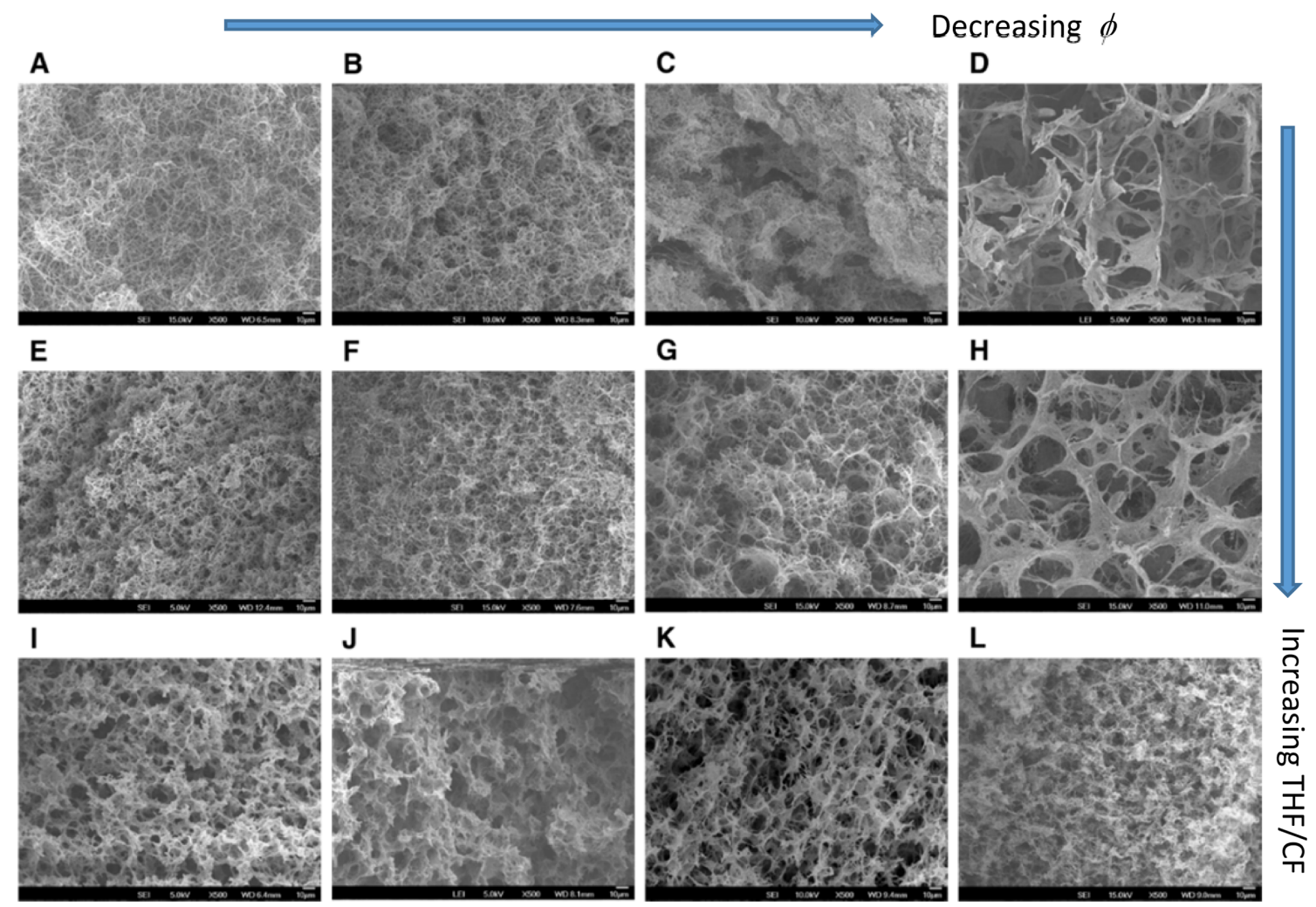

Figure 5. SEM images (500× magnification) of PHB scaffolds prepared from solutions of PHB in a mixture of CF and THF with varying THF/CF ratios and PHB concentrations. Scaffolds prepared from a fixed THF/CF ratio (30/70), and varying PHB concentrations $(\phi)$ of $(A) 0.03$, (B) 0.02 , (C) 0.01 , and (D) $0.005 \mathrm{~g} / \mathrm{mL}$. Scaffolds prepared from a fixed THF/CF ratio (43/57) and varying $\phi$ 's of (E) 0.03 , (F) 0.02, (G) 0.01, or (H) $0.005 \mathrm{~g} /$ $\mathrm{mL}$. Scaffolds prepared from a fixed THF/CF ratio (60/40), and varying $\phi$ 's of (I) $0.03,(\mathrm{~J}) 0.02$, (K) 0.01 , or (L) $0.005 \mathrm{~g} / \mathrm{mL}$.

phase but also increases the PHB concentration in the PHB-rich phase due to the decreased CF content. The larger polymer-poor phase can form micropores, and the congested environment of polymer-rich phase may inhibit nanofiber formation after drying. Here, the effect of polymer concentration on the morphology of scaffolds prepared by NIPS was investigated using SEM. Figure 5 shows SEM images of scaffolds with different THF/CF ratios and $\phi$ values. The microporosity increased with decreasing PHB concentration, regardless of the solvent composition. Similarly, the microporosity of polycarbonate scaffolds prepared by NIPS increased with decreasing polymer concentration. ${ }^{6}$ The volume ratio of the polymer-poor phase to the polymer-rich phase increased with decreasing $\phi$, which resulted in increased microporosity. As shown in Figure 5, the correlation between polymer concentration and nanofibrous morphology is rather complicated. Nanofibrous scaffolds (observed at THF/CF ratio of $30 / 70$ and 43/57) had a non-fibrous morphology at low polymer concentrations ( $\phi=0.005 \mathrm{~g} / \mathrm{mL}$, Figure 5(D), (H)). Similarly, the skeleton size of porous polycarbonate scaffolds prepared by NIPS became larger with decreasing polymer concentration. ${ }^{6}$ In contrast, as shown in Figure 5(I), 5(L), and Figure 6, at fixed THF/CF ratio of 60/40, the microporous scaffold with $\phi=0.005$ $\mathrm{g} / \mathrm{mL}$ displayed a more developed nanofiber morphology compared to that of scaffolds with higher $\phi$. The results indicates that the opimal $\phi$ range in which the polymer can form a well-defined nanofibrous morphology, shifted to a lower range of $\phi$ for THF/ CF ratio of 60/40 compared to THF/CF ratio of $30 / 70$ and 43/57. Different value of $\phi$ range for achieving a well-defined nanofi-
A

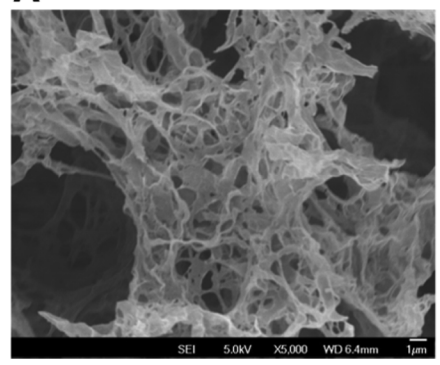

B

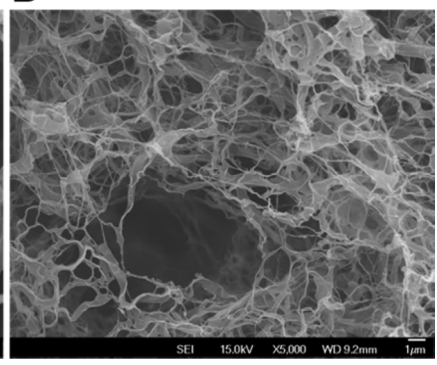

Figure 6. SEM images (5000× magnification) of PHB scaffolds with a fixed THF/CF ratio (60/40) and the PHB concentration ( $\phi$ ) of (A) $0.03 \mathrm{~g} /$ $\mathrm{mL}$ or (B) $0.005 \mathrm{~g} / \mathrm{mL}$.

brous morphology with different THF/CF ratio can be understood as follows. The scaffolds have the same $\phi$, but scaffolds with $\mathrm{THF} / \mathrm{CF}$ ratio of $60 / 40$ have a more congested environment of polymer-rich phases due to reduced $\mathrm{CF}$ content, which requires lower $\phi$ range to form nanofibers compared to that of the scaffolds with THF/CF ratio of $30 / 70$ and $43 / 57$. Here, it has been demonstrated that the morphology of PHB scaffolds obtained by NIPS largely depends upon the polymer concentration, as well as the solvent composition (ratio). Scaffolds formed at $\phi=0.005 \mathrm{~g} / \mathrm{mL}$ were not studied further because they were too mechanically weak to withstand a monolithic form.

The surface areas and mean pore diameters of the PHB scaffolds, which were determined from nitrogen adsorption-desorption isotherms and BET analysis, are summarized in Table 1. The specific surface area decreased when the PHB content was 
Table 1. Characteristic data of Brunauer-Emmett-Teller (BET) surface area and porosity of scaffolds prepared from a solution of PHB (concentration, $\phi$ ) in a mixture of THF and CF

\begin{tabular}{|c|c|c|c|c|c|c|c|c|c|}
\hline \multirow{3}{*}{$\begin{array}{c}\phi \\
(\mathrm{g} / \mathrm{mL})\end{array}$} & \multicolumn{9}{|c|}{ THF/CF (v/v) } \\
\hline & \multicolumn{3}{|c|}{$30 / 70$} & \multicolumn{3}{|c|}{$43 / 57$} & \multicolumn{3}{|c|}{$60 / 40$} \\
\hline & $\begin{array}{l}\text { Specific surface } \\
\text { area }\left(\mathrm{m}^{2} / \mathrm{g}\right)\end{array}$ & $\begin{array}{l}\text { Mean pore } \\
\text { size }(\mathrm{nm})\end{array}$ & $\begin{array}{c}\text { Porosity } \\
(\%)\end{array}$ & $\begin{array}{l}\text { Specific surface } \\
\text { area }\left(\mathrm{m}^{2} / \mathrm{g}\right)\end{array}$ & $\begin{array}{l}\text { Mean pore } \\
\text { size }(\mathrm{nm})\end{array}$ & $\begin{array}{c}\text { Porosity } \\
(\%)\end{array}$ & $\begin{array}{l}\text { Specific surface } \\
\text { area }\left(\mathrm{m}^{2} / \mathrm{g}\right)\end{array}$ & $\begin{array}{l}\text { Mean pore } \\
\text { size }(\mathrm{nm})\end{array}$ & $\begin{array}{l}\text { Porosity } \\
\text { (\%) }\end{array}$ \\
\hline 0.03 & $72.1 \pm 0.6$ & 9.8 & 97.0 & $68.4 \pm 0.5$ & 10.0 & 97.2 & $56.7 \pm 0.7$ & 11.9 & 97.0 \\
\hline 0.02 & $74.0 \pm 0.5$ & 10.9 & 98.3 & $46.8 \pm 0.6$ & 10.0 & 97.9 & $51.6 \pm 1.0$ & 16.0 & 98.1 \\
\hline 0.01 & $102.2 \pm 0.9$ & 8.9 & 99.1 & $13.6 \pm 0.6$ & 27.9 & 99.0 & $38.7 \pm 0.9$ & 13.8 & 98.9 \\
\hline
\end{tabular}

decreased to $0.01 \mathrm{~g} / \mathrm{mL}$ for scaffolds prepared from solutions at THF/CF ratios of $43 / 57$ and $60 / 40$, indicating that the loss of nanofibrous features is consistent with fewer nanofibers (Figure 5). In contrast, the surface area increased when PHB content was decreased to $0.01 \mathrm{~g} / \mathrm{mL}$ for scaffolds prepared from solutions at THF/CF ratio of 30/70. As can be seen in Figure 5(C), the well-defined nanofiber morphology was preserved despite the appearance of micropores. In fact, the nanofibers were thinner than at high $\phi$, resulting in an increased surface area of the scaffold. Microporosity and nanofibers are important features that can affect cell-scaffold interactions. NIPS can be used to easily adjust the scaffold microporosity and nanostructure of the scaffolds by varying polymer concentration and solvent composition.

\subsection{Mechanical properties of PHB scaffolds}

The compressive stress-strain curves of PHB scaffolds are shown in Figure S2. The compressive stress-strain curves of PHB scaffolds showed a linear elastic region at low strain and a non-linear plastic deformation plateau beyond the yield point. The initial linear elasticity for highly porous materials is associated with pore wall bending, and the long plateau in the stress-strain curves is related to the collapse of the pore wall. When the strain reached $60 \%$, the compressive stress increased rapidly, possibly because the pore walls touch each other and form solid bulks under increasing stress. The compressive modulus is derived from the slope of the initial linear portion of the stress-strain curve. The plateau stress is defined as the stress value at a strain of 0.2 . The effect of solvent composition and polymer concentration on the compressive moduli and plateau stress of PHB scaffolds is summarized in Figure 7. As discussed previously, the

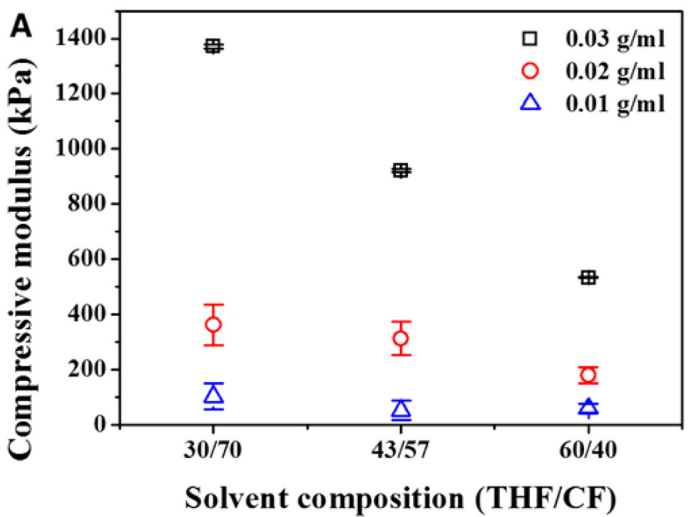

PHB scaffolds became mechanically weaker with increasing THF/ CF ratios. ${ }^{10}$ The absence of a nanofibrous structure was responsible for the decreased mechanical properties of the PHB scaffolds at higher THF/CF ratios. As the polymer concentration increased, PHB scaffolds became markedly stronger despite their similar porosities. In particular, the monolithic scaffolds at $\phi=0.01 \mathrm{~g} / \mathrm{mL}$ were too fragile to be utilized for cell proliferation. The dependence of solvent composition on the mechanical strength decreased with decreasing polymer concentration.

\subsection{Crystallization behavior of PHB scaffolds}

Differential scanning calorimetry (DSC) was performed on the PHB scaffolds to study the effect of PHB concentration on the crystalline state of the PHB scaffolds. During initial heating, a melting endotherm at a low temperature $\left(T_{\mathrm{m} 1}\right)$ of approximately $140^{\circ} \mathrm{C}$ was observed for all PHB scaffolds (Figure S3). This lower temperature melting endotherm was previously assigned to the intermediate, less-ordered crystalline phase induced by NIPS. ${ }^{10}$ The double-melting peaks observed in the higher temperature range of $160-180^{\circ} \mathrm{C}$ are known as the melting-recrystallization-melting of PHB during heating. The double-melting peaks did not show a clear correlation of peak temperature or enthalpy with polymer concentration and solvent composition or (Figure $\mathrm{S} 3$ and Table 2). However, $T_{\mathrm{m} 1}$ was found to be very sensitive to the PHB concentration, such that $T_{\mathrm{m} 1}$ shifted to lower temperatures at lower PHB concentration. The enthalpy $\left(\Delta H_{1}\right)$ of the melting peak of the intermediate, less-ordered crystalline phase also decreased with decreasing $\phi$ (Table 2). Decreased local concentration in the polymer-rich phase resulted in a lower degree of crystallization.

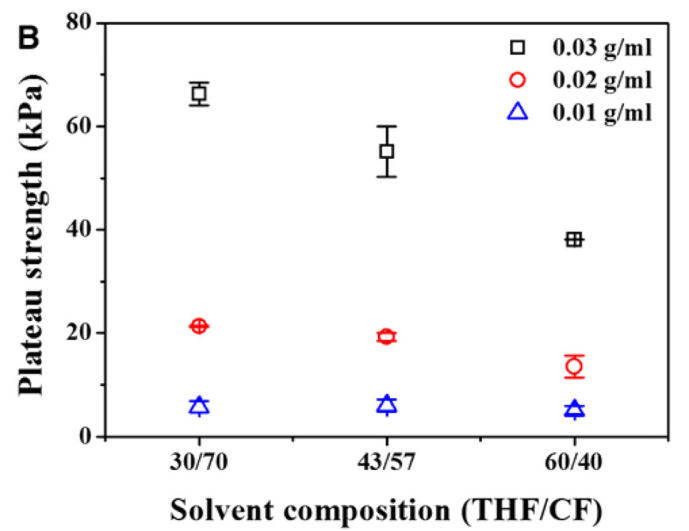

Figure 7. Mechanical properties of PHB scaffolds. (A) Compressive modulus and (B) plateau strength (at 0.2 strain) of PHB scaffolds prepared by NIPS with varying THF/CF ratios and $\phi$. The relative uncertainties (error bar) shown here are the standard deviations from multiple runs. 
Table 2. Enthalpy of the melting endothermic peaks for PHB scaffolds

\begin{tabular}{ccccc}
\hline$\phi(\mathrm{g} / \mathrm{mL})$ & $\mathrm{THF} / \mathrm{CF}(\mathrm{v} / \mathrm{v})$ & $\Delta H_{1}(\mathrm{~J} / \mathrm{g})$ & $\Delta H_{\mathrm{dm}}{ }^{a}(\mathrm{~J} / \mathrm{g})$ & $\Delta H_{\text {total }}(\mathrm{J} / \mathrm{g})$ \\
\hline \multirow{2}{*}{0.03} & $30 / 70$ & 28.6 & 68.4 & 97.0 \\
& $43 / 57$ & 17.7 & 77.4 & 95.1 \\
\hline \multirow{2}{*}{0.02} & $60 / 40$ & 12.8 & 65.6 & 98.4 \\
& $30 / 70$ & 18.5 & 74.9 & 79.2 \\
\hline \multirow{2}{*}{0.01} & $43 / 57$ & 16.6 & 75.1 & 77.5 \\
& $60 / 40$ & 2.5 & 56 & 76.0 \\
& $30 / 70$ & 20.0 & 67.8 & 82.7 \\
\end{tabular}

${ }^{a} \Delta H_{\mathrm{dm}}$ was calculated from double melting peaks and $\Delta H_{\mathrm{total}}=\Delta H_{1}+\Delta H_{\mathrm{dm}}$.

\subsection{Biocompatibility of HaCaT cells on the PHB scaffolds}

3-D biocompatible scaffolds play a vital role in cell-based tissue engineering. They serve as a temporary space for cell attachment and an extracellular matrix-like environment for cell growth and differentiation. HaCaT, an immortalized cell line of keratinocytes from adult skin, was used to examine if keratinocytes proliferate well on the surface of PHB scaffolds. A cell viability assay was used to evaluate the cytocompatibility of the scaffolds fabricated by NIPS. HaCaT seeded on PHB scaffolds were cultured for 3 days, and their viability was evaluated using the CCK-8 assay and SEM. Excellent viability of HaCaT cells was observed after a 3-day culture on all of the scaffolds (Figure 8). Tissue culture plates (control) induced less proliferation of HaCaT cells compared to that by the PHB scaffolds prepared by NIPS. High cell viability indicated that high-quality scaffolds with welldefined, uniform 3-D porous structures were successfully fabricated by NIPS using THF/CF. For scaffolds with $\phi=0.02 \mathrm{~g} / \mathrm{mL}$, there was no significant difference in cell viability with varying solvent composition (Figure 8(A)). It is important to note the improvement of mechanical properties for scaffolds with $\phi=$ $0.03 \mathrm{~g} / \mathrm{mL}$ compared to those with $\phi=0.02 \mathrm{~g} / \mathrm{mL}$ (Table 1, Figure 7). The increased mechanical strength and stiffness did not improve the cell viability of the nanofibrous scaffolds with THF/ CF ratio of 30/70 and 43/57 (Figure 8). However, microporous scaffolds with a THF/CF ratio of 60/40 showed markedly improved cell viability at higher $\phi(0.03 \mathrm{~g} / \mathrm{mL}$, Figure $8(\mathrm{~B}))$, indicating that the mechanical improvement of the scaffolds, significantly influences interactions between the microporous scaffolds and $\mathrm{HaCaT}$ cells.

The highest cell proliferation was observed for the PHB scaffold prepared by NIPS at THF $/ \mathrm{CF}$ ratio of $60 / 40$ and $\phi=0.03 \mathrm{~g} / \mathrm{mL}$. Interestingly, this scaffold did not display a nanofibrous morphology but had well-connected micropores. Furthermore, the absence of nanofibers led to a greatly reduced surface area and a poorer mechanical strength compared to those of scaffolds with lower THF/CF ratios. Many efforts have been made to construct nanofibrous scaffolds to mimic the extracellular matrix (ECM) for enhanced HaCaT cell proliferation. ${ }^{12,44-48}$ Nanofibrous scaffolds have excellent structural similarity to the ECM and provide a substratum for cell migration into defective sites. ${ }^{46}$ The importance of incorporating 3-D microporous structures into the scaffold for $\mathrm{HaCaT}$ cell proliferation has also been reported. ${ }^{49-52}$ The micropores in the scaffold can enable physical and biochemical communication between keratinocytes. Moreover, the micropores ensure a supply of active biological molecules, such as nutrients and growth factors, reach the cells from the cell culture medium. There has previously been no direct comparison of the effects of nanofibrous and microporous structure on cell viability. Our results indicate that the presence of nanofibers in scaffolds is not the most important parameter for HaCaT cell proliferation. Although the scaffolds with a nanofibrous morphology showed excellent proliferation, the positive effects due to scaffold microporosity were greater than the negative effects, e.g., smaller spe-
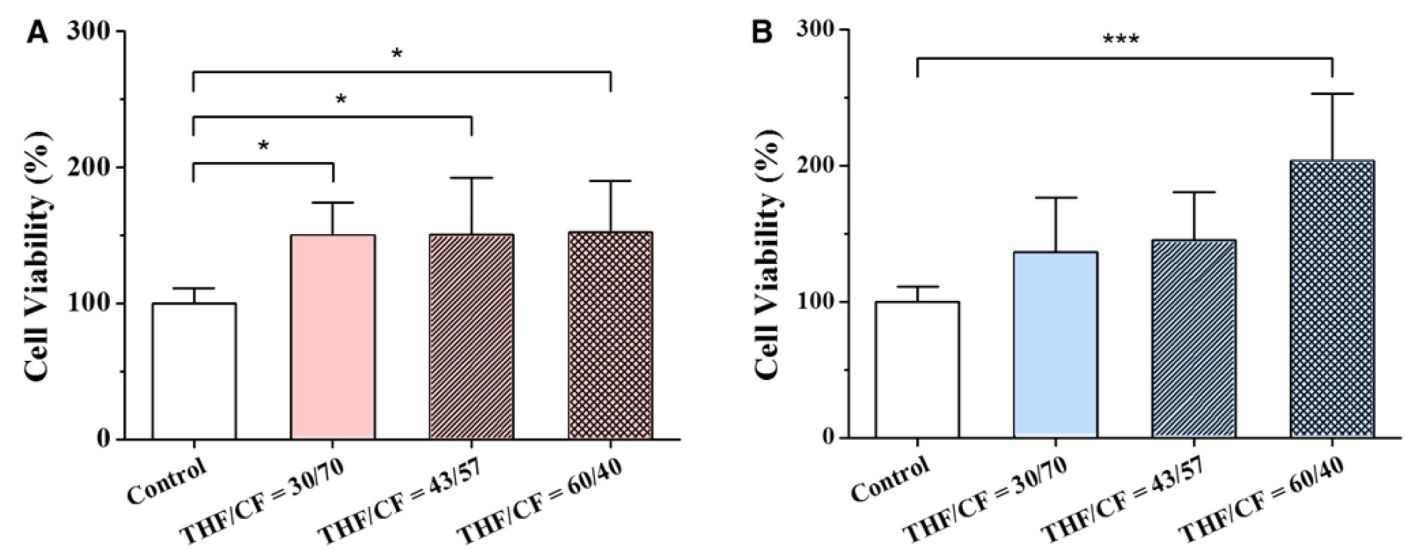

Figure 8. The cell viability of HaCaT on PHB scaffolds prepared from solutions with different THF/CF ratios and a fixed $\phi$ of (A) $0.02 \mathrm{~g} / \mathrm{mL}$ or (B) $0.03 \mathrm{~g} / \mathrm{mL}$. Statistical analysis was performed by a one-way AMOVA $\left({ }^{*} p<0.05,{ }^{* *} p<0.01\right.$, and $\left.{ }^{* * *} p<0.001\right)$. 
A

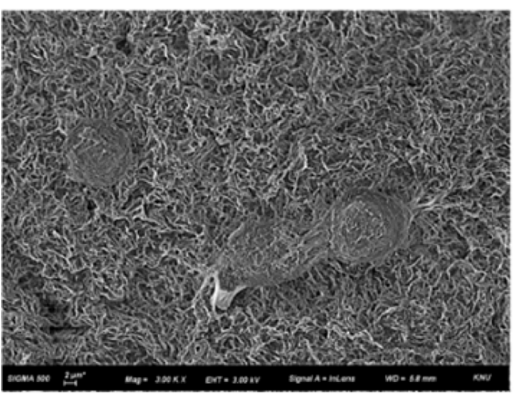

D

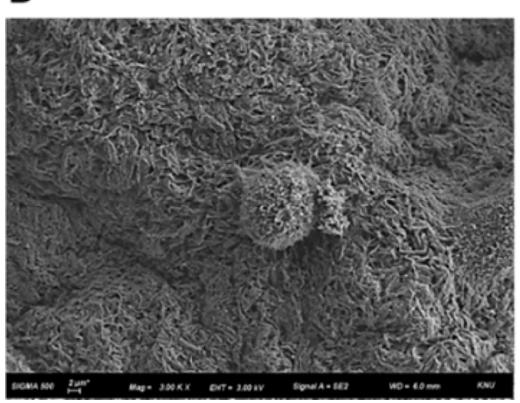

B

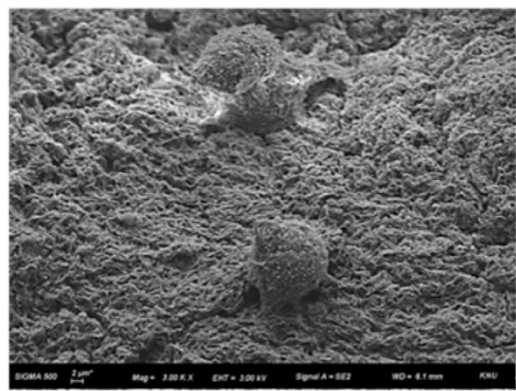

E

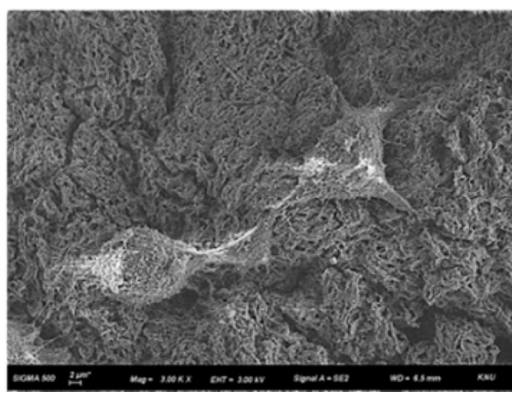

C

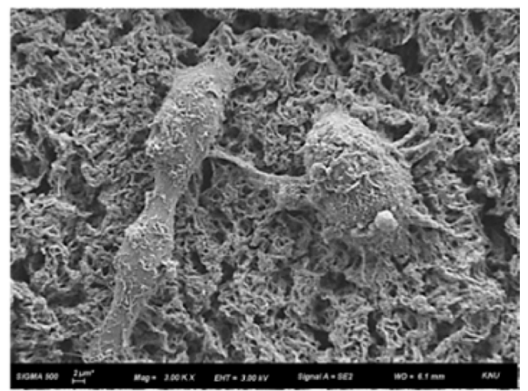

$\mathbf{F}$

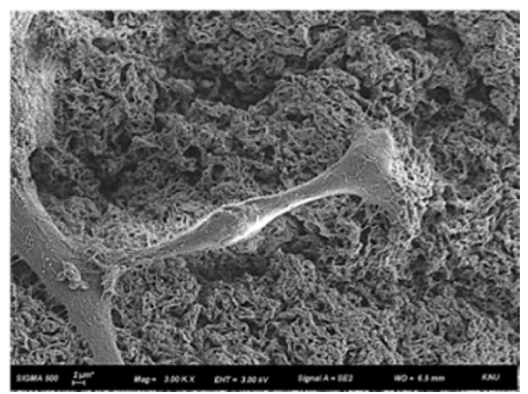

Figure 9. Representative SEM images showing the morphologies of HaCaT cells on PHB scaffolds prepared from solutions with different THF/CF ratios and $\phi$ values. Scaffolds were prepared either with a fixed $\phi$ of $0.02 \mathrm{~g} / \mathrm{mL}$ and the THF/CF ratio of (A) 30/70, (B) 43/57, or (C) 60/40 or with a fixed $\phi$ of $0.03 \mathrm{~g} / \mathrm{mL}$ and the THF/CF ratio of (D) 30/70, (E) 43/57, or (F) 60/40.

A

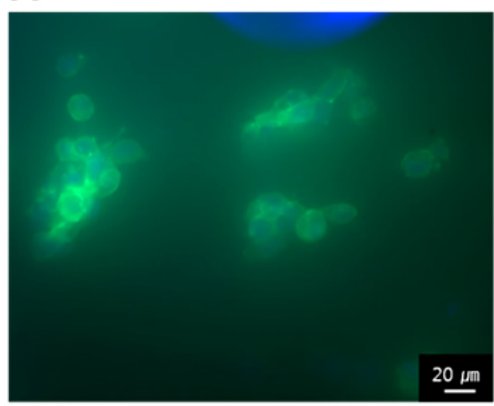

D

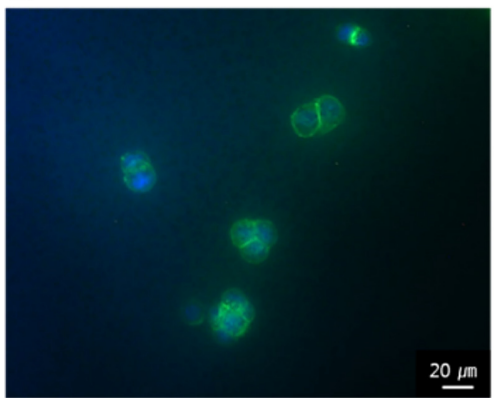

B

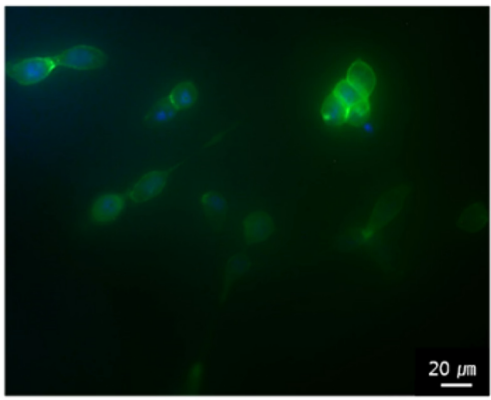

$\mathrm{E}$

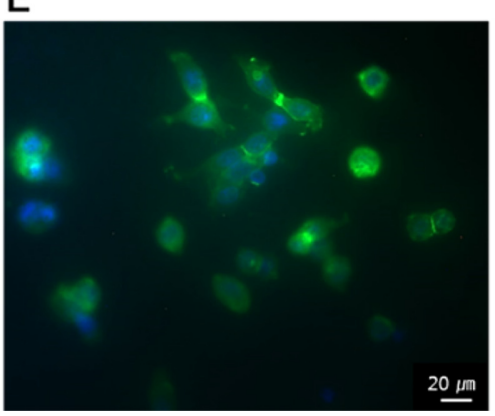

C

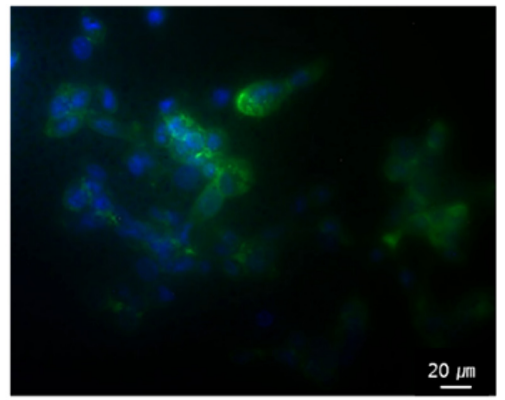

$\mathrm{F}$

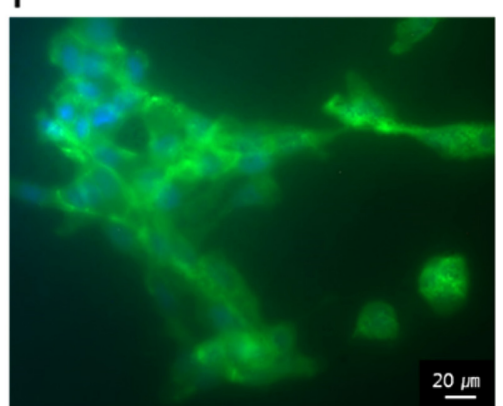

Figure 10. Representative immunofluorescence images of HaCaT on PHB scaffolds prepared from the solutions with varying THF/CF ratios and PHB concentrations. Scaffolds prepared either with a fixed PHB concentration ( $\phi$ ) of $0.02 \mathrm{~g} / \mathrm{mL}$ and the THF/CF ratio of (A) 30/70, (B) 43/57, or (C) $60 / 40$ or with a fixed $\phi$ of $0.03 \mathrm{~g} / \mathrm{mL}$ and the THF/CF ratio of (D) 30/70, (E) 43/57, or (F) 60/40. Cells were labeled by staining with Alexa Fluor 488 phalloidin (green) for actin filaments and DAPI (blue) for nuclei. Scale bar: $20 \mu \mathrm{m}$.

cific surface area and weaker mechanical strength.

The cell surface morphology and the actin cytoskeleton organization of HaCaT on the scaffolds were analyzed by SEM and immunofluorescence microscopy. The actin filaments of viable HaCaT that were cultured for 3 days showed a more stretched cell shape on PHB scaffolds prepared with THF/CF ratio of 60/
40 (Figure 9(C), (F)) compared to the less flattened and rounder cell shapes on nanofibrous scaffolds with lower THF/CF ratios (Figure 9(A), (B), (D), and (E)). The most organized actin cytoskeleton (good spreading and a high cell number) was observed on the scaffold with THF/CF ratio of $60 / 40$ and $\phi=0.03 \mathrm{~g} / \mathrm{mL}$ (Figure $10(\mathrm{~F})$ ), which agrees with viability tests (Figure 8). 


\section{Conclusions}

The present study demonstrated the influence of manufacturing parameters, such as polymer concentration and solvent mixture composition, on the morphology and properties of scaffolds made by NIPS. A quantitative investigation of the structure of PHB organogels and scaffolds over a wide length scale was performed by SANS analysis. The studied organogels had a complex hierarchical structure, extending over a wide range of length scales. The structural configuration on the micron length scale, in the low $Q$ range $<0.005 \AA^{-1}$, arose due to the presence of two separated phases: PHB-rich and PHB-poor. The SANS intensity of the PHB organogels was well described by Porod's law at high $Q$ values, $0.07<Q<0.1 \AA^{-1}$, indicating the presence of small crystallites in the polymer-rich phase. Fitting $I(Q)$ in the intermediate $Q$ range, $0.01<Q<0.035 \AA^{-1}$, with the $0 Z$ model provided the relationship between the fabrication parameters and the $\xi$ in the polymer-rich phase. Increasing the THF/CF ratio or $\phi$ effectively increased PHB concentration in the PHB-rich phase. SEM results indicate the opimal $\phi$ range in which the polymer can form a well-defined nanofibrous morphology, shifted to a lower $\phi$ range for THF/CF ratio of 60/40 compared to THF/CF ratio of $30 / 40$ and $43 / 57$. In vitro tests showed excellent proliferation of HaCaT cells on PHB scaffolds over 3 days. The HaCaT cells responded especially well to the PHB scaffolds made from solutions at THF/CF ratio of $60 / 40$ and $\phi=0.03 \mathrm{~g} / \mathrm{mL}$, showing good spreading and a high cell count. The present study found that scaffold microporosity is the most significant factor for improving HaCaT proliferation. Further work should be carried out on evaluating the biocompatibility of the scaffolds in relevant in vivo models.

Supporting information: Information is available regarding the photographs of PHB organogels and compressive stress-strain curves and DSC heating scans of PHB scaffolds. The materials are available via the Internet at http://www.springer.com/13233.

\section{References}

(1) M. Kobayashi, T. Yoshinori, T. Kozasa, K. Tashiro, J. Suzuki, S. Funahashi, and Y. Izumi, Macromolecules, 27, 1349 (1994).

(2) K.-Y. Lin, D.-M. Wang, and J.-Y. Lai, Macromolecules, 35, 6697 (2002).

(3) M. Muthukumar, Adv. Chem. Phys., 128, 1 (2004).

(4) X. Wang, H. Zhang, and S. C. Jana, J. Mater. Chem. A, 1, 13989 (2013).

(5) Q. Xing, X. Dong, R. Li, H. Yang, C. C. Han, and D. Wang, Polymer, 54, 5965 (2013).

(6) Y.Xin, T. Fujimoto, and H. Uyama, Polymer, 53, 2847 (2012).

(7) D. M. Wang and J. Y. Lai, Curr. Opin. Chem. Eng., 2, 229 (2013).

(8) E. Rezabeigi, P. M. Wood-Adams, and R. A. L. Drew, Polymer, 55, 6743 (2014).

(9) L. He, Y.Zhang, X. Zeng, D. Quan, S. Liao, Y.Zeng J. Lu, and S. Ramakrishna, Polymer, 50, 4128 (2009).

(10) J. Kang, H. Gi, R. Choe, and S. I. Yun, Polymer, 104, 61 (2016).

(11) A. Cesaro, D. Fabril, F. Sussichl, and G. Paradossi, Macromol. Symp., 138, 165 (1999).

(12) X.-T. Li, Y. Zhang, and G.-Q. Chen, Biomaterials, 29, 3720 (2008).

(13) K. S. Jack, S. Velayudhan, P. Luckman, M. Trau, L. Grøndahl, and J. Cooper-White, Acta Biomater, 5, 2657 (2009).

(14) X.-Y. Xu, X.-T. Li, S.-W. Peng, J.-F. Xiao, C. Liu, G. Fang, K. C. Chen, and G.-Q. Chen, Biomaterials, 31, 3967 (2010).

(15) M. T. Khorasani, S. A. Mirmohammadi, and S. Irani, Int. J. Polym. Mater.
Polym. Biomater., 60, 562 (2011).

(16) D. Guzman, H. Kirsebom, C. Solano, J. Quillaguaman, and R. HattiKaul, J. Bioact. Compat. Polym., 26, 452 (2011).

(17) K. M. Woo, V. J. Chen, and P. X. Ma, J. Biomed. Mater. Res. Part A, 67, 531 (2003).

(18) A. R. Boccaccini and V. Maquet, Compos. Sci. Technol, 63, 2417 (2003).

(19) X. Liu, L. A. Smith, J. Hu, and P. X. Ma, Biomaterials, 30, 2252 (2009).

(20) X. Liu and P. X. Ma, Biomaterials, 30, 4094 (2009).

(21) J.S. Chen, S.-L. Tu, and R.-Y.Tsay,J. Taiwan Inst. Chem. Eng., 41, 229 (2010).

(22) S.-H. Hsu, S. Huang, Y.-C. Wang, and Y.-C. Kuo, Acta Biomater., 9, 6915 (2013).

(23) A. Buzarovska, C. Gualandi, A. Parrilli, and M. Scandola, Compos. Part $B, 81,189$ (2015).

(24) J. Reuver, F. W. Altena, and C. A. Smolders, J. Polym. Sci. Part B: Polym. Phys., 24, 793 (1986).

(25) W. R. Burghard, L. Yilmaz, and A. J. McHugh, Polymer, 28, 2085 (1987).

(26) C. Daniel, M. D. Deluca, J.-M. Guenet, A. Brulet, and A. Menelle, Polymer, 37, 1273 (1996).

(27) C. He, W. Nie, and W. Feng, J. Mater. Chem. B, 2, 7828 (2014).

(28) E. Elmowafy, A. Abdal-Hay, A. Skouras, M. Tiboni, L. Casettari, and V. Guarino, Expert Rev. Med. Devic., 16, 467 (2019).

(29) Y. Ke, X. Y. Zhang, S. Ramakrishna, L. M. He, and G. Wu, Mater. Sci. Eng. C: Mater. Biol. Appl., 70, 1107 (2017).

(30) F. I. Butt, N. Muhammad, A. Hamid, M. Moniruzzaman, and F. Sharif, Int. J. Biol., Macromol., 120, 1294 (2018).

(31) N. Goonoo, A. Bhaw-Luximon, P. Passanha, S. R. Esteves, and D. Jhurry, J. Biomed. Mater. Res. Part B: Appl. Biomater., 105, 1667 (2017).

(32) J. Lim, M. You, J. Li, and Z. Li, Mater. Sci. Eng. C: Mater. Biol. Appl., 79, 917 (2017).

(33) B. Dariš and Ž. Knez, Acta Pharm., 70, 1 (2020).

(34) Q. Liu and G.-Q. Chen, J. Biomater. Sci. Polymer Edn., 19, 1521 (2008).

(35) Y. Ji, X.-T. Li, and G.-Q. Chen, Biomaterials, 29, 3807 (2008).

(36) D. Sundaramurthi, U. M. Krishnan, and S. Sethuraman,J. Biomed. Nanotechnol., 9, 1383 (2013).

(37) E. P. Gilbert, J. C. Schulz, and T.J. Noakes, Physica B, 385-386, 1180 (2006).

(38) S. R. Kline, J. Appl. Crystallogr., 39, 895 (2006).

(39) T. Kanaya, M. Ohkura, K. Kaji, M. Furusaka, and M. Misawa, Macromolecules, 27, 5609 (1994).

(40) T. Kanaya, M. Ohkura, H. Takeshita, K. Kaji, M. Furusaka, H. Yamaoka, and G. D. Wignall, Macromolecules, 28, 3168 (1995).

(41) H. Takeshita, T. Kanaya, K. Nishida, and K. Kaji, Physica B, 311, 78 (2002)

(42) R. J. Roe, in Methods of X-ray and Neutron Scattering in Polymer Science, Oxford University Press, 2000, pp 188-193.

(43) M. Shibayama, Soft Matter, 8, 8030 (2012).

(44) A. Neamnark, N. Sanchavanakit, P. Pavasant, R. Rujiravanit, and P. Supaphol, Eur. Polym. J., 44, 2060 (2008).

(45) B. Feng, H. C. Duan, W. Fu, Y. L. Cao, W. J. Zhang, and Z. Zhang, J. Biomed. Mater. Res., 103A, 431 (2015).

(46) G. Ramanathan, S. Singaravelu, M. D. Raja, N. Nagiah, P. Padmapriya, K. Ruban, K. Kaveri, T. S. Natarajan, U. T. Sivagnanam, and P. T. Perumal, RSC Adv., 6, 7914 (2016).

(47) S. Bhowmick, S. Rother, H. Zimmermann, P. S. Lee, S. Moeller, M. Schnabelrauch, V. Koul, R. Jordan, V. Hintze, and D. Scharnweber, Mater. Sci. Eng. C: Mater. Biol. Appl., 79, 15 (2017).

(48) M. Bacakova, J. Pajorova, D. stranska, D. Hadraba, F. Lopot, T. Riedel, E. Brynda, M. Zaloudkova, and L. Bacakova, Int.J. Nanomed, 12, 1143 (2017).

(49) J. Yang, G. Shi, J. Bei, S. Wang, Y. Cao, Q. Shang, G. Yang, and W. Wang, J. Biomed. Mater. Res., 62, 438 (2002).

(50) G. Peschel, H.-M. Dahse, A. Konrad, G. D. Wieland, P.-J. Mueller, D. P. Martin, and M. Roth, J. Biomed. Mater. Res., 85A, 1072 (2008).

(51) G. Ratheesh, J. R. Venugopal, A. Chinappan, H. Ezhilarasu, A. Sadiq, and S. Ramakrishna, ACS Biomater. Sci. Eng., 3, 1175 (2017).

(52) S. Khan, M. Ul-Islam, M. Ikram, S. U. Islam, M. W. Ullah, M. Israr, J. H. Jang, S. Yoon, and J. K. Park, Int. J. Biol., Macromol., 117, 1200 (2018).

Publisher's Note Springer Nature remains neutral with regard to jurisdictional claims in published maps and institutional affiliations. 